\title{
Clinical outcomes of prophylactic Damus-Kaye-Stansel anastomosis concomitant with bidirectional Glenn procedure
}

\author{
Masatoshi Shimada, MD, ${ }^{\mathrm{a}}$ Takaya Hoashi, MD, ${ }^{\mathrm{a}}$ Koji Kagisaki, MD, ${ }^{\mathrm{a}}$ Isao Shiraishi, MD, \\ Toshikatsu Yagihara, MD, ${ }^{a}$ and Hajime Ichikawa, MD $^{\mathrm{a}}$
}

Objective: We evaluated prophylactic Damus-Kaye-Stansel (DKS) anastomosis in association with the timing of a bidirectional Glenn (BDG) procedure as second-stage palliation aiming at Fontan completion to prevent late systemic ventricular outflow tract obstruction.

Methods: Between 1996 and 2005, 25 patients (14 boys; median age, 12 months) underwent a BDG procedure concomitant with DKS anastomosis. All had a systemic ventricular outflow tract through an intraventricular communication or morphologically developed subaortic conus and had previously undergone pulmonary artery banding. Enlargement of intraventricular communication and/or resection of a subaortic conus were not performed before or during the operation.

Results: Twenty-one ( $84 \%$ ) patients subsequently underwent a Fontan operation, with a follow-up period of 6.8 \pm 1.9 years (range, $4-11$ years), with no mortalities after the Fontan operation. Cardiac catheterization showed that systemic ventricular end-diastolic volume was significantly decreased from $187 \% \pm 74 \%$ of normal before BDG to $139 \% \pm 35 \%$ after $(P=.038)$ and to $73 \% \pm 14 \%$ at 4.3 years after the Fontan operation $(P<.001)$. However, the pressure gradient across the systemic ventricular outflow tract remained at $0.5 \pm 0.8 \mathrm{~mm} \mathrm{Hg}$ after DKS anastomosis and $0.6 \pm 2.3 \mathrm{~mm} \mathrm{Hg}$ at 4.6 years after the Fontan operation. None of the patients showed more than moderate aortic or neoaortic regurgitation, except 1 who progressed to pulmonary regurgitation after DKS anastomosis and required a reoperation for a systemic ventricular outflow tract. No anatomic properties affected late neoaortic valve function.

Conclusions: Regardless of a significant reduction in systemic ventricular volume, DKS anastomosis concomitant with a BDG procedure shows promise for a nonobstructive systemic ventricular outflow tract after a Fontan operation. (J Thorac Cardiovasc Surg 2012;143:137-43)

Supplemental material is available online.

In patients with a functionally single ventricle and specific anatomic properties, systemic ventricular outflow tract obstruction (SVOTO) can progress after reduction of systemic ventricular volume during staging for a Fontan strategy, resulting in an increase in systemic ventricular end-diastolic pressure and failure of Fontan circulation. ${ }^{1}$ One possible anatomic group has a systemic ventricular outflow tract (SVOT) through an intraventricular communication such as a ventricular septal defect (VSD) or bulboventricular foramen (BVF), whereas another has a morphologically developed subaortic conus.

\footnotetext{
From the Departments of Pediatric Cardiovascular Surgery and Pediatric

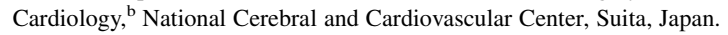
Disclosures: Authors have nothing to disclose with regard to commercial support. Read at the 91st Annual Meeting of The American Association for Thoracic Surgery, Philadelphia, Pennsylvania, May 7-11, 2011.

Received for publication May 8, 2011; revisions received July 13, 2011; accepted for publication Sept 15, 2011; available ahead of print Oct 13, 2011.

Address for reprints: Takaya Hoashi, MD, PhD, 5-7-1 Fujishiro-dai, Suita, Osaka, Japan 565-0873 (E-mail: thoashi@surg1.med.osaka-u.ac.jp). $0022-5223 / \$ 36.00$

Copyright (C) 2012 by The American Association for Thoracic Surgery doi:10.1016/j.jtcvs.2011.09.009
}

Representative surgical options for management of SVOTO are BVF/VSD enlargement and subaortic conus resection. However, those procedures may cause surgical heart block, ventricular dysfunction, and recurrent stenosis. Another option is application of a so-called Damus-KayeStansel (DKS) anastomosis, ${ }^{2-4}$ for which several technical modifications have been reported. The basic concept of these methods is use of both outflow tracts as a single systemic outflow by anastomosis between the main pulmonary trunk and ascending aorta. ${ }^{5}$

The midterm to long-term efficacy of DKS anastomosis is now widely recognized ${ }^{6-9}$; thus, the next issue is determination of optimal operative timing and anatomic indications. Since 1996, we have performed prophylactic DKS anastomosis concomitant with a bidirectional Glenn (BDG) procedure as a second-stage palliation procedure as part of a staging Fontan strategy to prevent late SVOTO. The purpose of the present study was to evaluate the clinical outcomes of DKS anastomosis performed concomitant with a BDG procedure.

\section{PATIENTS AND METHODS \\ Patients}

Between 1996 and 2005, 142 patients with a functionally single ventricle underwent a BDG procedure at our institution. Of those, 25 (14 boys 


$\begin{aligned} & \text { Abbreviations and Acronyms } \\ & \text { BDG }=\text { bidirectional Glenn } \\ & \mathrm{BVF}=\text { bulboventricular foramen } \\ & \mathrm{DKS}=\text { Damus-Kaye-Stansel } \\ & \mathrm{PA}=\text { pulmonary artery } \\ & \mathrm{SVOT}=\text { systemic ventricular outflow tract } \\ & \mathrm{SVOTO}= \text { systemic ventricular outflow tract } \\ & \text { obstruction } \\ & \% \mathrm{VOT}= \text { ventricular outflow tract indexed by the } \\ & \text { normal value } \\ & \mathrm{VSD}= \text { ventricular septal defect }\end{aligned}$

and 11 girls) underwent concomitant DKS anastomosis in a side-by-side fashion and were followed up for more than 5 years (Table 1). The median age and body weight at the operation were 12 months old (range, 5-120 months) and $7.6 \mathrm{~kg}$ (range, $3.8-27.5 \mathrm{~kg}$ ), respectively. This study was approved by the National Cerebral and Cardiovascular Center Institutional Review Board.

All patients underwent pulmonary artery (PA) banding to regulate pulmonary high blood flow before the BDG procedure. Banding tape was placed just below the pulmonary bifurcation during PA banding to avoid injury to the pulmonary valve. Including these 25 patients, a total of 47 patients underwent PA banding for single-ventricle physiology during the same period. Four of 22 patients, including 3 with atrial isomerism and total anomalous pulmonary venous connection, died before the second-stage BDG. In the remaining 18 patients, SVOTO did not develop during the study period.

Eleven of 25 patients had previously undergone repair of coarctation of the aorta or an interrupted aortic arch. No patients underwent enlargement of the VSD or BVF nor subaortic conus resection before or during the operation. Since 2003, we have been anastomosing a very short expanded polytetrafluoroethylene tube to the inferior wall of the right and/or left PA for use as a "pouch" as preparation for a subsequent Fontan operation without cardiopulmonary bypass. ${ }^{10}$

\section{Anatomic Properties for Prophylactic DKS Procedure}

On the basis of our institutional experience and previous reports, we identified 2 anatomic properties to designate patients at high risk for SVOTO during staging for a Fontan strategy or after Fontan completion (Table 2). The first group of patients had a SVOT through the VSD or BVF. In these, the aorta usually arises from a rudimentary or hypoplastic ventricle (Figure E1, $A$ ). The second group had a morphologically developed subaortic conus (Figure E1, B).

\section{Operative Procedures}

All patients underwent DKS in a side-by-side fashion without additional patch materials to augment the anastomosis. ${ }^{5,7}$ In brief, the proximal great arteries were transected at the level of the PA band above the sinuses. The facing walls of the proximal great arteries were incised in a V shape and then anastomosed, after which end-to-end anastomosis of the distal ascending aorta was performed and a ventricular outlet created.

In the past, we performed end-to-side anastomosis in some patients, although semilunar valve dysfunction sometimes occurred owing to enlargement of the sinus of Valsalva, which might be caused by turbulent flow and distortion of the sinus itself. Now, we use a side-by-side anastomosis to maintain the shape of the native sinus of Valsalva and obtain a straight blood flow stream to preserve the function of the semilunar valve. ${ }^{11}$
TABLE 1. Patient characteristics

\begin{tabular}{lc}
\hline Male/female (n) & $14: 11$ \\
Age (mo) at operation, median (range) & $12(5-120)$ \\
Body weight (kg) at operation, median (range) & $7.6(3.8-27.5)$ \\
Previous PA banding (n) & $25 / 25$ \\
Previous CoA/IAA repair (n) & $11 / 25$ \\
Method of BDG (n) & \\
BDG & 21 \\
Bilateral BDG & 3 \\
Total cavopulmonary shunt (Kawashima) & 1 \\
Concomitant procedures (n) & \\
ASD creation/enlargement & 17 \\
PA plasty & 12 \\
Atrioventricular valve repair & 6 \\
Placement of PA pouch & 6 \\
VSD/BFV enlargement & 0 \\
Subaortic conus resection & 0 \\
\hline
\end{tabular}

PA, Pulmonary artery; $C O A$, coarctation of the aorta; IAA, interrupted aortic arch; $B D G$, bidirectional Glenn procedure; $A S D$, atrial septal defect; $V S D$, ventricular septal defect; $B V F$, bulboventricular foramen.

\section{Study Method}

We retrospectively evaluated patient records, as well as catheter and echocardiography reports, in regard to the following variables: (1) overall results: cumulative survival, rate of Fontan completion, and freedom from reoperation for SVOTO; (2) serial changes in systemic ventricular end-diastolic pressure and percentage of normal systemic ventricular end-diastolic volume measured by a catheter examination before and after BDG with DKS and $4.3 \pm 1.5$ years after the Fontan operation; and (3) postoperative semilunar valve function estimated by echocardiographic findings obtained at $5.0 \pm 1.3$ years after the Fontan operation.

\section{Statistical Analysis}

Data are expressed as the mean \pm standard deviation or the median (range), as appropriate, and were analyzed using SPSS software (SPSS Institute, Inc, Chicago, Ill). Actuarial survival and freedom from reoperation for SVOTO were estimated using the Kaplan-Meier method.

TABLE 2. Anatomic indications

\begin{tabular}{lc}
\hline & No. \\
\hline A. SVOT through VSD/BVF & \\
1. TA or severe TS with VA discordant & 5 \\
2. MA or severe MS with normal AV and VA connection & 1 \\
3. DILV with rudimentary RV and VA discordant & 3 \\
4. Hypoplastic LV with VA concordant & 4 \\
5. DORV or VA discordant and balanced two ventricles & 4 \\
with unsuitable VSD(s) for biventricular repair & \\
B. Morphologically developed subaortic conus & 4 \\
1. MA and DORV & 3 \\
2. DIRV with rudimentary LV and DORV & 1 \\
3. DORV with hypoplastic LV & \\
\hline
\end{tabular}

SVOT, Systemic ventricular outflow tract; $V S D$, ventricular septal defect; $B V F$, bulboventricular foramen; $T A$, tricuspid atresia; $T S$, tricuspid stenosis; $V A$, ventriculoatrial; $M A$, mitral atresia; $M S$, mitral stenosis; $A V$, atrioventricular; $D I L V$, double-inlet left ventricle; $R V$, right ventricle; $L V$, left ventricle; $D O R V$, double-outlet right ventricle; $V S D$, ventricular septal defect. 

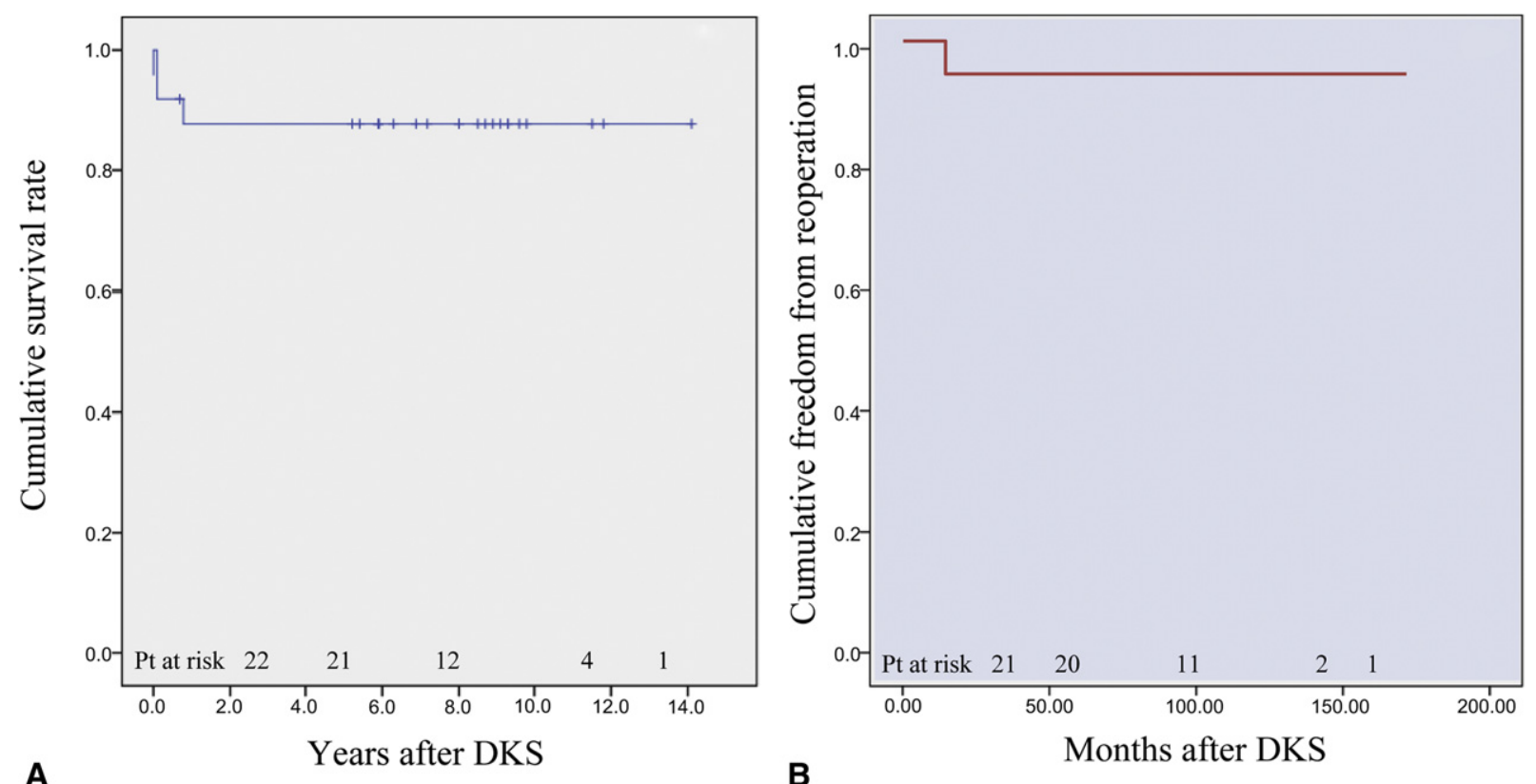

FIGURE 1. A, Cumulative survival. B, Freedom from reoperation for systemic ventricular outflow tract (Kaplan-Meier method). DKS, Damus-Kaye-Stansel.

\section{RESULTS \\ Overall Outcomes}

The mean follow-up period after DKS and the Fontan operation were $7.0 \pm 3.6$ years $(0-14$ years $)$ and $6.8 \pm 1.9$ years (4-11 years), respectively. The overall survival at 10 years after DKS was $87.7 \%$ (Figure 1, $A$ ). There were 2 early deaths, 1 from cerebral hemorrhage at 20 days after the operation and 1 from low-output syndrome at 18 days after the operation. In addition, 1 late death occurred owing to arrhythmia at 10 months after the operation. None of the patients who died showed SVOTO or semilunar valve regurgitation in follow-up examinations.

Twenty-one $(84 \%)$ patients subsequently underwent Fontan completion (Figure 2), of whom 15 (71\%) underwent the Fontan operation without cardiopulmonary bypass. There were no deaths during or after Fontan completion.

Freedom from reoperation is as reflective of physician behavior as of the underlying disease, and its rate at 10 years after DKS was $95.5 \%$ (Figure $1, B$ ). Only 1 patient, who had pulmonary regurgitation after DKS anastomosis, required a reoperation for neoaortic regurgitation 11 months after DKS and none required a reoperation for SVOTO.

\section{Hemodynamic Analysis}

Cardiac catheter examinations before BDG concomitant with the DKS procedure were performed in all patients. Furthermore, post-Fontan catheter examinations were performed in all patients at $4.3 \pm 1.5$ years after Fontan completion. The mean systemic ventricular end-diastolic volume decreased from $187 \% \pm 74 \%$ of normal before
BDG with DKS to $139 \% \pm 35 \%$ of normal at 6.8 months after BDG with DKS $(P=.038)$, then to $73 \% \pm 14 \%$ of normal at 4.3 years after the Fontan operation $(P<.001)$ (Figure 3,A). The mean pressure gradient across the SVOT was $7.7 \pm 10 \mathrm{~mm} \mathrm{Hg}$ (range, $0-35 \mathrm{~mm} \mathrm{Hg}$ ) before BDG and DKS. Five patients showed a pressure gradient greater than $10 \mathrm{~mm} \mathrm{Hg}$ across the SVOT. After BDG and DKS, the mean pressure gradient across the SVOT was $0.5 \pm 0.8 \mathrm{~mm} \mathrm{Hg}$ (range, $0-2 \mathrm{~mm} \mathrm{Hg}$ ), which remained until 4.3 years after the Fontan operation (Figure 3, B).

\section{Semilunar Valve Insufficiency}

Postoperative echocardiographic analysis was performed in 22 long-term survivors at $5.0 \pm 1.3$ years after DKS.

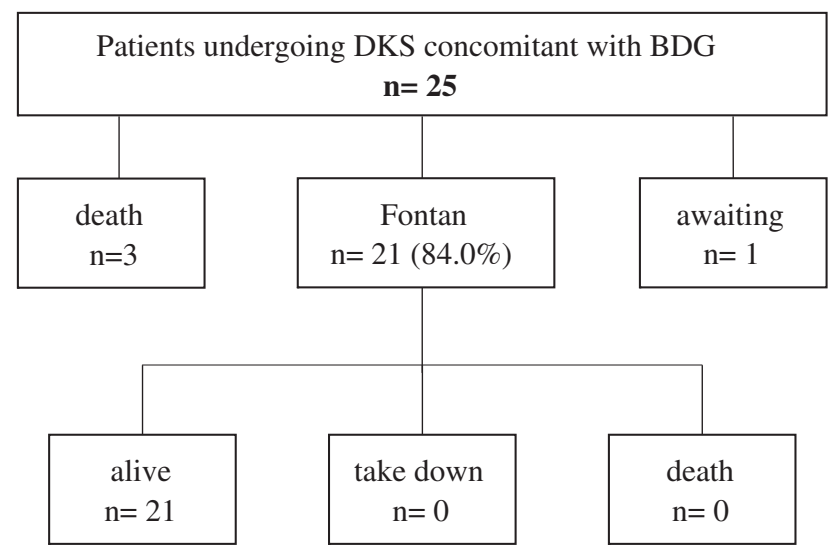

FIGURE 2. Rate of Fontan completion after bidirectional Glenn $(B D G$ procedure and Damus-Kaye-Stansel (DKS) anastomosis. 

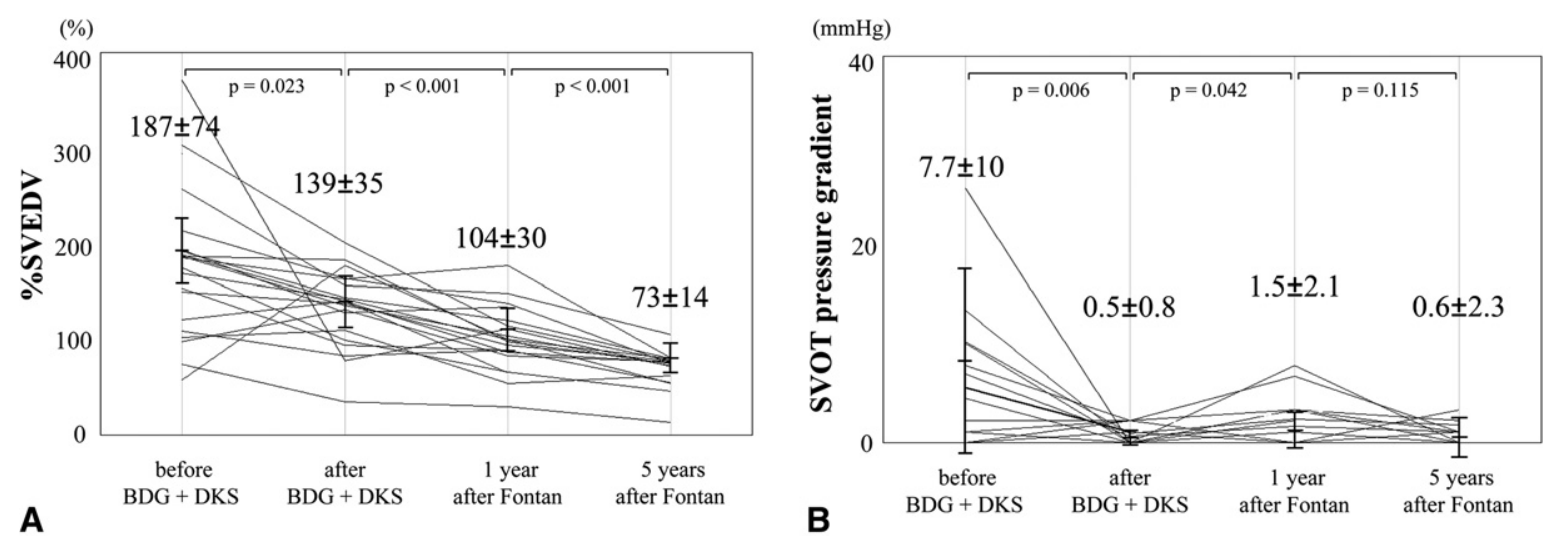

FIGURE 3. Serial changes of mean systemic ventricular end-diastolic volume (A) and mean pressure gradient across the systemic ventricular outflow tract (B). SVEDV, Systemic ventricular end-diastolic volume; SVOT, systemic ventricular outflow tract.

Aortic valve regurgitation was absent in 14 patients and mild in 8 , whereas neoaortic regurgitation was absent in 19 patients and mild in $3 ; 1$ patient underwent valve replacement, as noted earlier.

\section{DISCUSSION}

The present findings demonstrate that prophylactic DKS anastomosis concomitant with a BDG procedure is promising for a nonobstructive SVOT, even after a significant reduction in systemic ventricular volume provided by staged palliative operations and a Fontan operation. Eighty-four percent of our patients subsequently underwent a Fontan procedure, and greater than moderate aortic or neoaortic regurgitation was observed in only 1 patient during the follow-up period.

An important finding of the present study is that systemic ventricular volume after BDG is reduced to about three quarters of that before BDG and then continues reducing to approximately $70 \%$ of normal about 5 years after a Fontan operation. Thus, even if SVOTO is not clinically evident before BDG, specific anatomic groups are at risk for late SVOTO. As shown in our study, the first group has an SVOT through an intraventricular communication such as from a VSD or BVF. ${ }^{12,13}$ In this group, the size of the VSD or BVF can be restrictive, as ventricular volume is reduced. Although enlargement of the intraventricular communication might be effective to overcome this problem, ${ }^{13,14}$ it also includes potential risk of surgical heart block. The second group has a morphologically developed subaortic conus. Although resection of that might be effective, such a procedure will frequently result in inadequate relief and require a reoperation or injure the septal perforator branch, which causes dysfunction of the anterior interventricular septum.

Next, the optimal timing of prophylactic DKS during the course of a staged Fontan operation is a matter of concern. Inasmuch as cardiopulmonary bypass and cardioplegic arrest are essential for a DKS procedure, they are better performed at the time of BDG or the Fontan operation to avoid another open cardiac operation. Furthermore, systemic ventricular preload reaches a minimal level after BDG without additional pulmonary blood flow. Indeed, we found that systemic ventricular volume after BDG was reduced to $75 \%$ of that before BDG. Thus, we recommend that prophylactic DKS be performed at the time of BDG. The other advantage of DKS concomitant with BDG is to minimize the cardiopulmonary bypass time during the Fontan operation. It is widely known that extended cardiopulmonary bypass time is a significant risk factor for a Fontan procedure, although occurrence of enteropathy causing a loss of protein after the Fontan operation is also an important concern. ${ }^{15,16}$ Therefore, we perform a Fontan operation without cardiopulmonary bypass for selected patients,,${ }^{10}$ and DKS at the time of BDG plays a role as a preparative procedure for off-pump Fontan completion.

Preceding PA banding has been reported to affect semilunar valvular function after DKS anastomosis. ${ }^{11,17,18}$ In our series, 1 patient required pulmonary valve replacement for severe pulmonary regurgitation after DKS and BDG. This patient previously underwent PA banding at another hospital, at which time banding tape was placed on the level of the sinotubular junction, which forced us to distort the pulmonary sinus of Valsalva at the time of DKS anastomosis. This was the reason for development of neoaortic regurgitation soon after DKS and BDG. Thus, care should be taken regarding the timing of PA banding so as to not to create a deformity of the pulmonary sinus for its later use as a systemic semilunar valve. Presently, we are careful to place banding tape on the main PA as distally as possible. Except for this single case, the functions of both semilunar valves functioned well in our patients until late after the Fontan operation. We also consider that DKS used for side-by-side anastomosis of the great arteries above the sinotubular junction helps to 
maintain the shape of the native sinus of Valsalva and preserve the functions of the semilunar valves.

Complication by an obstructive aortic arch is another widely known risk factor for late left ventricular outflow tract obstruction. Indeed, approximately half of the patients in this cohort had undergone surgery for an interrupted aortic arch or coarctation before the DKS/BDG procedures. DKS or a modified Norwood with a shunt is an alternative strategy to treat these patients, as reported by Bradley and colleagues. ${ }^{19}$ However, we prefer to avoid cardiopulmonary bypass during the neonatal period if possible. For the same reason, we select such a procedure for only those patients who show significant SVOTO before PA banding, whereas it is always performed during the young infantile period after initial bilateral PA banding with the arterial duct being left open.

\section{Study Limitations}

Prediction of late SVOTO during staging for a Fontan procedure or after Fontan completion is still a matter of concern. We did not determine the indication for prophylactic DKS based on the absolute size of SVOT or BVF, but rather anatomic properties, as listed in Table 2. Thus, some of the patients might have been free from late SVOTO without DKS.

Matitiau and colleague ${ }^{20}$ measured BVF size in 28 neonates and infants with tricuspid atresia or a double-inlet left ventricle and transposition of the great arteries. Eight were free from late SVOTO, including the only 4 patients who survived after Fontan completion. They found that late obstruction was unlikely if the BVF area index was greater than $2 \mathrm{~cm}^{2} / \mathrm{m}^{2}$. Also, Miura and colleagues ${ }^{8}$ clearly demonstrated serial changes in the diameter of the ventricular outflow tract indexed by the normal value (\%VOT) before PA banding, after PA banding, after BDG, and after Fontan in patients with functionally single ventricle and transposition of the great arteries or double-outlet right ventricle. DKS for relief of SVOTO was necessitated in all patients who had completion of a Fontan operation, except for 1 with a $\%$ VOT of $153 \%$ before PA banding. They concluded that $\%$ VOT before PA banding less than $120 \%$ was a risk factor for late SVOTO after PA banding.

As described in both of the aforementioned studies, we are aware that there are patients in whom SVOTO does not develop in the future, even if high-risk anatomic properties are present. However, it is also true that late SVOTO is quite prevalent and can be fatal when it occurs under Fontan circulation. The other issue is that the exact timing of measurement of the ventricular outflow tract or BVF after Fontan completion was not described in either study. Thus, SVOTO might develop later in those patients, because ventricular volume continues to decline for at least 5 years after Fontan completion, as we found in the present study. Considering these findings and that it is ethically impossible to randomize patients to BDG with or without DKS, our prophylactic strategy is considered to be justified.

In conclusion, regardless of significant ventricular volume reduction in patients with an anatomy indicating possible ventricular outflow obstruction, prophylactic DKS anastomosis concomitant with BDG resulted in nonobstructed SVOT late after the Fontan operation.

\section{References}

1. Finta KM, Beekman RH, Lupinetti FM, Bove EL. Systemic ventricular outflow obstruction progresses after the Fontan operation. Ann Thorac Surg. 1994;58: 1108-12.

2. Damus PS. Correspondence. Ann Thorac Surg. 1975;20:724-5.

3. Kaye MP. Anatomic correction of transposition of great arteries. Mayo Clin Proc. 1975;50:638-40

4. Stansel HC Jr. A new operation for d-loop transposition of the great vessels. Ann Thorac Surg. 1975;19:565-7.

5. Waldman JD, Lamberti JJ, George L, Kirkpatrick SE, Mathewson JW, Spicer RL, et al. Experience with Damus procedure. Circulation. 1988;78(5 Pt 2):III32-9.

6. DeLeon SY, Ilbawi MN, Tubeszewski K, Wilson WR Jr, Idriss FS. The DamusStansel-Kaye procedure: anatomical determinants and modifications. Ann Thorac Surg. 1991;52:680-7.

7. Lamberti JJ, Mainwaring RD, Waldman JD, George L, Mathewson JW Spicer RL, et al. The Damus-Fontan procedure. Ann Thorac Surg. 1991;52 676-9.

8. Miura T, Kishimoto H, Kawata H, Hata M, Hoashi T, Nakajima T. Management of univentricular heart with systemic ventricular outflow obstruction by pulmonary artery banding and Damus-Kaye-Stansel operation. Ann Thorac Surg. 2004 77:23-8.

9. Hiramatsu T, Imai Y, Kurosawa H, Takanashi Y, Aoki M, Shinoka T, et al. Midterm results of surgical treatment of systemic ventricular outflow obstruction in Fontan patients. Ann Thorac Surg. 2002;73:855-60.

10. Shiraishi S, Uemura H, Kagisaki K, Koh M, Yagihara T, Kitamura S. The offpump Fontan procedure by simply cross-clamping the inferior caval vein. Ann Thorac Surg. 2005;79:2083-7.

11. Fujii Y, Kasahara S, Kotani Y, Takagaki M, Arai S, Otsuki S, et al. Double-barre Damus-Kaye-Stansel operation is better than end-to-side Damus-Kaye-Stansel operation for preserving the pulmonary valve function: the importance of preserving the shape of the pulmonary sinus. J Thorac Cardiovasc Surg. 2011; 141:193-9.

12. Freedom RM. Subaortic obstruction and the Fontan operation. Ann Thorac Surg 1998;66:649-52

13. Cerillo AG, Murzi B, Giusti S, Crucean A, Redaelli S, Vanini V. Pulmonary artery banding and ventricular septal defect enlargement in patients with univentricular atrioventricular connection and the aorta originating from an incomplete ventricle. Eur J Cardiothorac Surg. 2002;22:192-9.

14. Cheung HC, Lincoln C, Anderson RH, Ho SY, Shinebourne EA, Pallides S, et al Options for surgical repair in hearts with univentricular atrioventricular connection and subaortic stenosis. J Thorac Cardiovasc Surg. 1990;100:672-81.

15. Mosca RS, Kulik TJ, Goldberg CS, Vermilion RP, Charpie JR, Crowley DC, et al. Early results of the Fontan procedure in one hundred consecutive patients with hypoplastic left heart syndrome. J Thorac Cardiovasc Surg. 2000;119:1110-8.

16. Powell AJ, Gauvreau K, Jenkins KJ, Blume ED, Mayer JE, Lock JE. Perioperative risk factors for development of protein-losing enteropathy following a Fontan procedure. Am J Cardiol. 2001;88:1206-9.

17. Huddleston CB, Canter CE, Spray TL. Damus-Kaye-Stansel with cavopulmonary connection for single ventricle and subaortic obstruction. Ann Thorac Surg. 1993;55:339-45.

18. Amin Z, Backer CL, Duffy CE, Mavroudis C. Does banding the pulmonary artery affect pulmonary valve function after the Damus-Kaye-Stansel operation? Ann Thorac Surg. 1998;66:836-41.

19. Bradley SM, Simsic JM, Atz AM, Dorman BH. The infant with single ventricle and excessive pulmonary blood flow: results of a strategy of pulmonary artery division and shunt. Ann Thorac Surg. 2002;74:805-10.

20. Matitiau A, Geva T, Colan SD, Sluysmans T, Parness IA, Spevak PJ, et al. Bulboventricular foramen size in infants with double-inlet left ventricle or tricuspid atresia with transposed great arteries: influence on initial palliative operation and rate of growth. J Am Coll Cardiol. 1992;19:142-8. 


\section{Discussion}

Dr Robert D. Jaquiss (Durham, NC). I appreciate the opportunity to discuss this paper, which describes the clinical course of 25 children with univentricular heart and originally unrestricted pulmonary blood flow with the potential for SVOTO. Patients were treated with initial PA banding, often in conjunction with other procedures, subsequently with a BDG shunt and the inventive PA pouch, followed finally by a Fontan completion, which in a majority of patients was actually accomplished off pump. There were 3 deaths between the BDG-DKS operation and the subsequent Fontan procedure, and late follow-up shows no SVOTO, excellent Fontan hemodynamics, and preserved semilunar valve function. I enjoyed the presentation, which is Dr Shimada's first international presentation, and I congratulate him on that. I am also grateful to him and his colleagues for sending me a copy of the manuscript well in advance of the meeting. I have 3 questions for him.

The first relates to PA banding, which is not a benign procedure. In fact, the mortality rate in a recent report from another center in Osaka described 6 deaths in 21 patients with similar anatomy undergoing PA banding. An alternative strategy, which has been advocated by some, particularly in the setting of coexistent arch obstruction, is to proceed with an initial shunt and perform the DKS operation at the original operation as advocated by Scott Bradley's group from South Carolina several years ago.

My first question concerns the original cohort, which led to the 25 patients described in this report. How many patients actually underwent initial PA banding for this anatomy? Did all of them survive and did they all get to this combination DKS and BDG operation?

Dr Shimada. Thank you very much for a very important question. During the same period to this study, 47 patients underwent PA banding for single-ventricle physiology, excluding bilateral PA banding for hypoplastic left heart syndrome and its variant.

A total of 4 deaths were observed. There were 2 operative or inhospital deaths after PA banding. Two interstage deaths before the BDG shunt occurred. Three of these patients had right isomerism with total anomalous pulmonary venous connection.

Among the rest of the 43 patients, Fontan completion was done in 35, with 5 patients waiting for BDG and 2 waiting for Fontan. There was 1 late death after Fontan completion in a patient who had right isomerism with total anomalous pulmonary venous connection.

Dr Jaquiss. My next question relates to the fact that not all patients with the aorta arising from a rudimentary ventricle or a subaortic conus will eventually have outflow tract obstruction, and so the approach that has been advocated here is a very aggressive one. In the prior study from the other center at Osaka, the group attempted to identify predictors of subsequent outflow tract obstruction and suggested a selective application of this approach that you have described here. Do you now advocate a very aggressive combination BDG and DKS in all patients with this anatomy regardless of whether they have outflow tract obstruction? In your presentation today, about half of the patients actually had no obstruction at the time of the DKS, so this is indeed very aggressive.

Dr Shimada. We did not apply the same method of measuring the ventricular outflow tract. In cases including patients classified into the second group, we decided the indication for DKS when the ventriculogram showed a possible ventricular outflow tract obstruction after reduction of ventricular volume or when a significant pressure gradient was detected. We have not quantified this possibility yet. This is generally true if the conus septum is largely deviated and aligned to the ventricular septum. When the conus septum is not aligned to the ventricular septum, such as is the case with side-by-side great vessel relationships, DKS is unlikely to be indicated.

In our series, all patients had undergone PA banding. As previously reported, it has been widely recognized that PA banding as a first palliation can be a cause of subaortic stenosis. To some extent, PA banding might be causing subaortic stenosis. Of course, some surgeon may prefer DKS with a Blalock-Taussig shunt in the neonatal period. However, we prefer to avoid cardiopulmonary bypass during the neonatal period if possible.

Dr Jaquiss. My last question is a simple technical one. I have trouble sometimes doing this operation that you have described without using some sort of patch. Your diagram seemed to show that you do this with an entirely autologous approach, that is, no pericardium, polytetrafluoroethylene (Gore-Tex patch; W.L. Gore \& Associates, Inc, Flagstaff, Ariz), or homograft. Is your diagram correct that you do this with entirely autologous material?

Dr Shimada. We have performed double-barrel-shaped reconstruction in all these patients only with autologous tissue. We think it is better in terms of somatic growth.

Talking about the anastomosis method, in the past we had performed end-to-side anastomosis in some patients. However, among these patients, semilunar valve dysfunction occurred owing to an enlargement of the sinus of Valsalva, which might be caused by turbulent flow and the distortion of the sinus itself. Since then, we choose the side-by-side anastomosis to maintain the shape of the native sinus of Valsalva and obtain straight blood flow stream to preserve the semilunar valve's function.

Also, preceding PA banding is reported to affect semilunar valve function after the DKS anastomosis. In this study, 1 patient required pulmonary valve replacement for his severe pulmonary regurgitation after DKS and BDG. In this case, the PA banding tape was placed at the level of sinotubular junction in another institution without regard for the next DKS anastomosis. This might cause distortion of the pulmonary sinus of Valsalva at the time of DKS anastomosis. Now we pay attention when placing the banding tape on the main PA as distally as possible so as not to make a deformity of the pulmonary sinus for the later use of this as a systemic semilunar valve.

Dr Jaquiss. Thank you very much. Again, I congratulate you and your coauthors on this excellent series and a very clear presentation.

Dr Hisashi Nikaidoh (Tulsa, Okla). It is a wonderful presentation and I really think it is a great series.

You already mentioned the importance of maintaining the integrity of semilunar valve anatomy in performing this particular operation. I agree, so I will not go into that discussion.

However, I would like to look at your approach not as a prophylactic operation but more like a plan. It is coming anyway, so you are going to do it. I think we could change the wording to the "planned concomitant DKS."

The question I have is that one of the very important indicators of such occurrence, the left ventricular outflow obstruction or ventricular outlet obstruction, is the presence of coarctation. How 
often have you seen coarctation among this particular group of patients?

Dr Shimada. In this series, 11 patients have coexistence of coarctation of the aorta. There were no significant differences between group A and group B in 11 patients.

Dr Shunji Sano (Okayama, Japan). I just have a comment that we now very aggressively perform DKS anastomosis. As Dr Nikaidoh said, it is not prophylactic. It is a planned operation at the time of initial palliation or BDG or at the Fontan, any time, even if the patient has no gradient across the left ventricular outflow tract, because in the long-term, left ventricular outflow tract obstruction may have developed. Even if there is no obstruction, I think there is an energy loss through the ventricle to the kinked aorta.

I have just 1 question. Did any patient require a DKS concomitant with Fontan who had no left ventricular tract obstruction at the timing of banding and BDG?

Dr Shimada. Six patients underwent DKS at the time of the Fontan operation. 

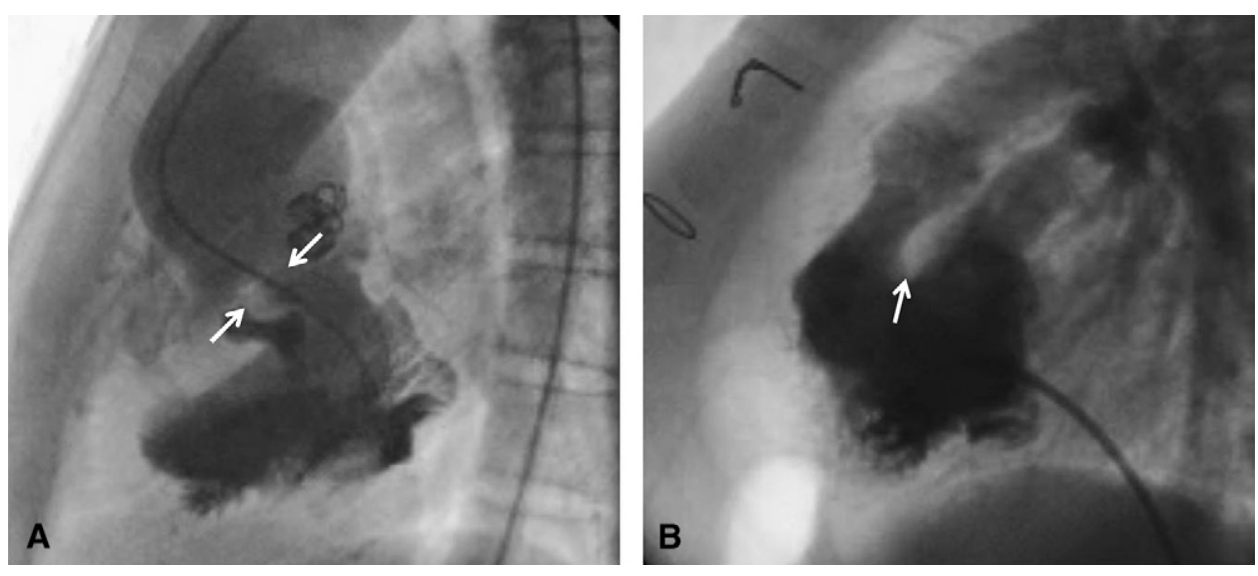

FIGURE E1. Angiography showing a systemic ventricular outflow tract through a restrictive ventricular septal defect (white arrows) in a patient with a double-inlet left ventricle, rudimentary right ventricle, and L-malposition of the great arteries (A) and a morphologically developed subaortic conus (white arrow) in a patient with a double-inlet left ventricle, double-outlet right ventricle, and D-malposition of the great arteries (B). 